\title{
¿̇a jubilación como derecho? Los debates que los Planes de Inclusión Previsional dejaron pendientes
}

\author{
Retirement as a right? \\ Debates that Pension Inclusion Plans left unfinished
}

\section{PALABRAS CLAVE}

Inclusión previsional

Construcción de sentidos

Derechos

Ciudadanía

\section{KEYWORDS}

Retirement inclusion

Construction of meaning

Rights

Citizenship

\section{RESUMEN}

En este artículo presentaremos algunos aspectos de una de las políticas públicas de la década pasada que, creemos, se encuentra entre las que más capacidad efectiva de inclusión, extensión de derechos y construcción de igualdad ha tenido: los Planes de Inclusión Previsional (PIP). A partir de la reseña de una investigación cualitativa desarrollada entre los años 2014 y 2015, reconstruiremos algunos de los sentidos que los propios destinatarios de esta política imprimieron a su experiencia. Trataremos de dimensionar, desde sus relatos en primera persona, los modos en los que estos jubilados y jubiladas se apropiaron simbólicamente de su novedosa condición de sujetos con derecho a la protección social. Finalmente, abordaremos algunas tensiones relacionadas a los sentidos sedimentados acerca de lo previsional y su contraposición frente a una semántica distinta de las políticas sociales en clave de derechos y ciudadanías. ¿Quiénes serían, en la Argentina que se configuraba en la primer década y media del siglo XXI, los adultos mayores con derecho a tener este derecho? Veremos que los discursos de los destinatarios de la jubilación por moratoria no arrojaron -al momento de la investigación, por lo menos- una respuesta unívoca, dando pistas de los múltiples debates que los PIP dejaron abiertos. Ahora, dos años después de aquel trabajo de campo y en medio de una vorágine de cambios en materia política y económica -además de en materia previsional- bien vale la pena revisitar aquellas cuestiones pendientes.

\section{ABSTRACT}

In this article we analyse some aspects of one of the last decade public policy which we characterize as one of those with the greatest capacity of inclusion, spreading of rights and construction of equality: the Pension Inclusion Plans (PIP). By starting from a qualitative research developed between 2014 and 2015, we reconstruct some of the meanings that this policy recipients stamped on their experience. We analyse from the point of view of pensioners, the way they symbolically appropriated their new status as subjects entitled to social protection. Finally, we approach some tensions related to the sedimented meanings about the pensions and its contradiction to a different semantics of social policies in terms of rights and citizenship. Who would be, in the first decade and a half of the XXI century argentine, the older adults with the right to have this right? We see that the retires answers were not univocal being a sample of the many debates that the PIP left open. Now, two years after that field work and in the midst of deep changes in political and economic matters - as well as in pension matters - it is worth revisiting those outstanding issues.

Fecha de recibido: 07/08/2017 | Fecha de aceptado: 11/10/2017 | Fecha de publicado: 28/12/2017

Cita sugerida: Corsiglia Mura, L. (2017). ¿La jubilación como derecho? Los debates que los Planes de Inclusión Previsional dejaron pendientes. Cuestiones de Sociología, 17, e041. https://doi.org/10.24215/23468904e041 


\section{Introducción}

En este artículo vamos a presentar algunos aspectos de una de las políticas públicas de la década pasada que, creemos, ha estado entre las más efectivas en capacidad de inclusión y extensión de derechos: los Planes de Inclusión Previsional. Aunque sin ser nombrados desde su inicio explícitamente de ese modo, los que después se llamarían Planes de Inclusión Previsional (PIP) ${ }^{1}$, nacieron hacia fines de 2004como una solución acotada ante los graves indicadores de vulnerabilidad social que afectaban a las poblaciones de adultos mayores. Sin embargo, por diversos motivos terminaron deviniendo en una política clave que, de conjunto con una batería más amplia de intervenciones en materia previsional, transformaron cuantitativa y cualitativamente la estructura del sistema de jubilaciones y pensiones de nuestro país.

Por supuesto, esta política no puede ser pensada por fuera de un clima de época que, hacia principios de Siglo XXI, y en el contexto más general de la región, reconstruiría una semántica diferente acerca de los roles del Estado como agente de reparación de anteriores agravios provocados por décadas de políticas neoliberales ${ }^{2}$.En ese marco, la redefinición de políticas sociales -dentro de las que se incluye a las políticas previsionales-incorporarían la dimensión de derechos, inclusión, equidad; así como paulatinamente iría avanzando la dimensión de la universalidad $\underline{3}$ como objetivo deseable.

Tal el caso de la política que aquí recorreremos que, fundamentalmente a través de dos moratorias previsionales de amplísimas facilidades de acceso, extendió la cobertura jubilatoria a más de tres millones y medio de personas ${ }^{4}$. Esta medida duplicó en aproximadamente dos años la cantidad de prestaciones vigentes, incorporando al ejercicio del derecho a la jubilación a poblaciones que habían permanecido tradicionalmente afuera de los sistemas de la seguridad social. Esta incorporación, por su parte, se daría en condiciones de absoluta igualdad al resto de los jubilados, salvando el detalle de que, durante los primeros cinco años de percepción del haber, se descontaría la cuota de la moratoria.

El resultado de los PIP, de la mano del efecto de los reiterados incrementos de las jubilaciones mínimas, de la posterior Ley de Movilidad y de las modificaciones en torno a la administración y lógica del sistema previsional, generaría múltiples planteos (teóricos, mediáticos y políticos), mayormente relacionados a la esfera económica. Sin embargo, menos extendidos serían los debates en torno a las dimensiones políticas y simbólicas en juego. Y justamente sobre este último punto versará el presente artículo.

Reconstruiremos aquí los sentidos que los propios destinatarios imprimieron a esta política. A partir de sus relatos, trataremos de dimensionar los modos en los que estos jubilados y jubiladas se apropiaron simbólicamente de su novedosa condición.

Este tipo de análisis nos parece indispensable para desnaturalizar los imaginarios que socialmente circulan acerca de las nociones de protección social, de derechos, de universalidad, en una sociedad con un acelerado proceso de envejecimiento poblacional y un sostenido estado de desprotección de una porción importante de su población. ¿Quiénes son los sujetos -adultos mayores en nuestro caso- con derecho a ser socialmente sostenidos?

Hay diferentes ámbitos donde buscar respuestas a esa pregunta. Aquí, empezamos por las 
percepciones de los propios destinatarios de una política que expandió masivamente los límites de la inclusión y la igualdad.

Los datos que se presentan corresponden al resultado de una investigación realizada entre los años 2014 y 2015 para obtener el título de Especialista en Políticas Sociales. Y si bien la política específica investigada ha sido actualmente discontinuada y reemplazada en parte por una pensión universal no contributiva (una nueva política previsional, inserta en un nuevo clima de época que está en plena configuración), la cuestión del derecho a la jubilación sigue estando en el centro de las agendas públicas. Y esto, justamente porque lo que subyace a la discusión sobre los derechos previsionales, no es en última instancia otra cosa que una pregunta por la comunidad, por la ciudadanía.

\section{Lo político de las políticas sociales: una mirada epistemológica que atañe a la política previsional}

La configuración de un escenario político novedoso a inicios del Siglo XXI abrió en la región, y en nuestro país en particular, un clima de época contrario a los cánones neoliberales, lo que obligó a la revisión de algunos de los marcos epistemológicos que habían imperado desde los años 90 a la hora de pensar al Estado y a las políticas. En esta nueva configuración de lo estatal, las políticas sociales -continente dentro del que debemos incorporar a las políticas previsionales- tendrán un importante rol en la construcción de los nuevos discursos acerca de cómo se concibe el orden de lo social, sus contornos, los sujetos con o sin derechos, los dispositivos de inclusión o exclusión. Como dicen Andrenacci y Repetto, es necesario asociara la política social "con los modos con que las sociedades diseñan las formas de pertenencia de los sujetos" entendiendo con esto los "procesos de inscripción de los sujetos en la organización social a través de la cual se obtiene no sólo 'un lugar' físico y simbólico, sino también el derecho a ser sostenido" (Andrenacci y Repetto, 2006, p. 46). Y en ese sentido, si acordamos en que las políticas sociales marcan los límites yacuerdos políticos en torno a la desigualdad socialmente tolerable en cada tiempo histórico, los Planes de Inclusión Previsional construyen un punto unívoco de referencia para el retroceso de desigualdades en la población de adultos mayores.

Así, en un marco de transformación de los sentidos simbólico-políticos de lo estatal, y de clara reformulación de las matrices argumentales de las políticas sociales ${ }^{\underline{5}}$, consideraremos a $\operatorname{los}$ PIP como una de las intervenciones más radicales a la hora de pensar las transformaciones dentro del campo de la protección social $\underline{6}$.

Antes de detenernos en la especificidad de los PIP, recuperaremos parte de la historia de la previsión social en nuestro país, destacando algunos de los ejes rectores de sus lógicas de funcionamiento y sus matrices legitimatorias.

\section{Historia, lógica y sentidos comunes del sistema previsional argentino.}

Los sistemas previsionales se encuentran dentro de la esfera más amplia de la seguridad social e incluyen al conjunto de coberturas de contingencias ante la vejez, invalidez y muerte (Fiscella, 2005). Podríamos simplificar diciendo que la discusión sobre los diferentes modelos de previsión 
social hace principalmente eje en resolver algún tipo de mecanismo que asegure ingresos a los adultos mayores. Y esto, claro, nos remite a sociedades que dan por supuesto un eje de integración social trabajo-céntrico ${ }^{7}$. Ahora bien, los modos de resolver esta cuestión y sus marcos legitimatorios son variados, tanto de una sociedad a otra, como dentro de una misma sociedad, a lo largo del tiempo.

De los dos modelos paradigmáticos principales de sistemas previsionales, el contributivo y el no contributivo $^{-}$, en nuestro país se aplicó principalmente el primero. Obedeciendo a la lógica del Seguro Social, se fundó en la idea de un seguro a la vejez para los trabajadores, colocando a la contribución (del mismo trabajador, así como del empleador o eventualmente también del Estado) en el eje tanto de la financiación, como de la legitimación de la protección. Esta manera de concebir lo previsional se constituyó en un sentido común consolidado a lo largo de la historia, en el que aparecería como casi evidente que la jubilación resultaba una suerte de ingreso dinerario relacionado a la anterior condición salarial de los sujetos. Baste con mencionar la capacidad de interpelación que tiene la consigna del $82 \%$ móvil, para darnos una idea de hasta qué punto este sentido es dominante ${ }^{9}$.

De este modo, a la hora de explicar el acceso a la jubilación, el trabajo será la primera credencial considerada como legítima, aunque convivirá, en una ambigüedad permanentemente, con la credencial de los aportes. Como corolario, cristalizará una noción de reconocimiento de la jubilación como derecho, no de todos los sujetos a partir de determinada edad, sino solamente para los trabajadores. Pero tampoco para todos los trabajadores, sino específicamente para aquellos que hayan realizado aportes a lo largo de sus trayectorias activas. Es decir, un derecho solo para los que tuvieran recorridos laborales continuados al interior del mercado formal de trabajo. En este esquema, la resolución social de las contingencias a la vejez no solo presupone la existencia de una sociedad trabajo-céntrica, sino que, además, requiere de la sostenibilidad y constancia en el tiempo de un mercado de trabajo altamente expansivo y formal. Veremos prontamente, estos anhelos respecto al mercado de trabajo no tardarían en entrar en crisis.

Así, el sistema previsional de nuestro país fue de los primeros de la región. Nació a principios de siglo $\mathrm{XX}^{10}$ y se extendió masivamente a los trabajadores activos de todas las ramas laborales en el marco de los primeros dos gobiernos peronistas $\underline{11}$. Por su parte, recién fue unificado en criterios hacia fines de los años 60, manteniéndose hasta los años 90 bajo administración estatal y con la lógica de reparto y solidaridad intergeneracional. Si bien tendría desde su inicio un fuerte sesgo corporativo-evidenciando mejores condiciones a aquellos actores sindicales con mayor poder de negociación- y su canal de acceso se mantendría atado a la trayectoria laboral de los sujetos, hasta la década del 70 -en una sociedad que aún mantenía una amplia expansión del empleo asalariadose consolidaría como un sistema con niveles relativamente altos de cobertura $\frac{12}{}$. Esta característica se iría erosionando a lo largo de los años 80, retrocediendo la cantidad de personas cubiertas, así como la calidad de la prestación previsional brindada. Finalmente, hacia los años 90, la semiprivatización del sistema ${ }^{13}$ modificaría los parámetros estructurales de lo previsional, al introducir la administración privada a la seguridad social y reformular los criterios de legitimación simbólica del derecho a la jubilación. A partir de la reforma neoliberal, el sesgo hacia el pilar de 
capitalización haría cristalizar un sentido común que ahora asociaría cada vez más el derecho a la jubilación al pago de aportes en clave individual, debilitando los criterios de solidaridad horizontal e intergeneracional anteriormente hegemónicos. En esta etapa, producto de las modificaciones sociales estructurales aplicadas, de la brutal alteración del mercado de trabajo, y de las modificaciones paramétricas del sistema previsional en sí mismo $\frac{14}{}$, se reducirían drásticamente los niveles de cobertura $\frac{15}{}$. A partir de la crisis de 2001 y la salida devaluatoria de inicios de 2002, el sistema previsional no hizo sino erosionarse aún más.

De este modo el ingreso al siglo XXI encontraría una población cada vez más envejecida $\frac{16}{}$, con sus integrantes adultos mayores cada vez menos protegidos. Para el 2000, habrá apenas poco más del $50 \%$ de las personas en edad de jubilarse accediendo a esta prestación (Arza, 2004, citado en Lo Vuolo, 2007; Danani y Beccaria, 2011). A esto hay que sumarle un contexto general que contabilizaba para 2002 una tasa de desempleo cercana al 20\%, un 49\% de trabajadores sin aportes y más del $50 \%$ de la población en condiciones de pobreza (ANSES, 2010).

Vemos entonces que a lo largo del primer siglo del sistema previsional distintas modificaciones se fueron implementando. El formato de capitalización, o el solidario; la capacidad de cobertura; el cálculo de la prestación y la capacidad y fuente de financiamiento; la administración pública, o la administración privada o mixta. Diversos debates han cruzado la historia de lo previsional en Argentina, derivando en diferentes reformas con alto impacto en la concepción de este derecho. Sin embargo, hay un elemento atado a las definiciones primigenias de su modelo paradigmático que ha cristalizado en una suerte de sentido común indiscutible: la concepción de la jubilación como un derivado de la trayectoria laboral de las personas. Más aún, de su capacidad de aportes a lo largo de su vida activa $\underline{17}$.

Ahora bien, justamente estos criterios sedimentados serían los que comenzarían a crujir ante la emergencia social de principios de siglo XXI y la evidencia de que el sistema, tal como estaba, excluía a gran parte de la población potencialmente destinataria.

Es aquí donde resulta oportuno retomar la idea de que la definición de previsión social lleva implícita una cierta concepción de lo social y de la ciudadanía. Ya dijimos que la dimensión del derecho a la jubilación no es extensible -en la formulación históricamente sedimentada en nuestro país- a todas las personas que, a partir de determinada edad, quedan dificultadas para resolver autónomamente sus medios económicos de supervivencia. Ahora bien, su concepción asociada al trabajo y los aportes tuvo gran efectividad mientras parecían realizables las "utopías del desarrollismo" que, hasta medidos de los años 70, pensaban posible una sociedad "relativamente homogénea, con pleno empleo, estabilidad social, familias nucleares y crecimiento económico sostenido" (Dvotskin, 2017, p. 5).

Sin embargo, hecho añicos ese sueño, el sistema previsional no hacía más que evidenciarla existencia dramática de una frontera (cada vez más profunda) entre adultos mayores con derecho y otros arrojados a los avatares de su propia capacidad (individual o familiar) para resolver la supervivencia. Una obviedad: nuestra sociedad está atravesada por múltiples exclusiones y desigualdades que, lejos de extinguirse como por arte de magia al final del ciclo vital, suelen potenciarse como acumulación de desventajas. En ese sentido Fleury (2006) utiliza la categoría de 
exclusión ${ }^{\underline{18}}$ para pensar en una desigual distribución de recursos y poderes, en una dimensión mucho más amplia que la mera cuestión de ingresos. Una cuestión de "esfera pública restringida que no incluye a una gran parte de la población" (Fleury,2006, p. 109). En similar sintonía Arditi (2007, p. 125) habla de "ciudadanías de geometría variable" dando cuenta de las accesibilidades diferenciales de grupos e individuos a la ciudadanía.

Tanto para Arditi (2007) como para Jelin (2011), Mouffe (1998) o Fleury (2002, 2006) la ciudadanía y los sujetos comprendidos por ella son categorías que se definen de modo histórico y contingente y a partir de lo propiamente conflictivo del orden de lo político, donde la comunidad (y su pertenencia) aparecen como algo disputado y en continua redefinición. Y es ahí donde nos interesa inscribir las transformaciones de los sentidos de la estatalidad, y de las lógicas legitimantes de las políticas sociales y previsionales.

Fleury (2002) enfatiza la relación entre la noción de ciudadanía y las políticas sociales, y explicita que estas últimas requieren de acuerdos sociales acerca de la desigualdad o la igualdad tolerable, implican definiciones acerca de los grupos con los que la sociedad acepta (o no) ser solidaria y establece los niveles de sacrificio colectivo deseables para la promoción social. Estas cuestiones aparecieron en discusión, aunque más no fuere de modo fáctico, a partir de la emergencia social de fin de siglo XX. La crítica situación social y el brutal impacto que esto tenía, entre otras cosas, en las poblaciones de adultos mayores, posibilitaría que las fronteras de demarcación entre quiénes se entendía que debían ser protegidos y quiénes no, comenzaran a ser puestas en cuestión, flexibilizadas y, en algunos casos, radicalmente modificadas.

A partir de 2002, pero mucho más profundamente a partir de 2003, se darían una serie de medidas dentro de las políticas previsionales que Danani y Beccaria (2011, p. 117) y Danani e Hintze (2011 y 2013) van a presentar como un quiebre en la dirección del sistema o, más enfáticamente, como una contra-reforma (Danani y Beccaria, 2011, p. 136).Este conjunto de políticas va a alterar las lógicas de lo que hasta el momento eran considerados los límites de la inclusión/exclusión en materia de seguridad social.

Haciendo un punteo cronológico de estas modificaciones encontramos que a partir de 2002 comenzarán a registrarse aumentos de haberes por decreto ${ }^{19}$. Estos aumentos serán proporcionalmente más importantes para las escalas más bajas, generando rápidamente un proceso de achatamiento de la pirámide de haberes a partir de una redistribución vertical de ingresos $\underline{20}$. En 2005 entrará en vigencia la Ley de Jubilación Anticipada, que incorporará en uno de sus artículos la posibilidad de adhesión a la moratoria que configurará lo que posteriormente se denominará como Plan de Inclusión Previsional. Esta Ley posibilitará el ingreso de más de 2.5 millones de nuevos jubilados y jubiladas, alcanzando temporalmente niveles de cobertura superiores al 95\%.En 2007 se sancionará la Ley de Libre Opción $\underline{21}$, y en 2008 la Ley de Movilidad Jubilatoria establecerá un nuevo mecanismo de actualización de haberes para todos los tramos. Finalmente, en diciembre de 2008 terminaría de darse un giro copernicano en el Sistema Previsional con la sanción de la Ley 26.425 de Creación del SIPA (Sistema Integrado Previsional Argentino), deshaciendo el modelo de administración mixta y restituyendo la modalidad de reparto, de solidaridad intergeneracional y de administración estatal. Hacia fines de 2014, con la sanción de una nueva Ley de Inclusión 
Previsional, se habilitaría otra moratoria con vigencia hasta octubre de 2016 a través de la que se buscaría sostener los niveles de cobertura previsional cercanos a la universalidad. Finalmente, en 2016, nuevas y radicales transformaciones impactarán en el sistema previsional, pero ahora con sentidos que en gran medida resultan inversos a los anteriores. Estas modificaciones abren un nuevo ciclo, sobre el que será necesario desarrollar nuevas indagaciones.

Pero volviendo al período que aquí estamos analizando, diremos que las medidas que van desde 2002 hasta el cierre de la Segunda Ley de Moratoria en 2016 han puesto en discusión no sólo el rol del Estado como agente de las políticas, sino también el lugar de los sujetos en tanto merecedores de la protección social. En primer lugar, la política de aumentos diferenciales para los tramos más bajos de haberes suavizó la lógica de estamentación atada a la capacidad contributiva. Enmarcado en un contexto de crisis social en la que los adultos mayores ocupaban un lugar de vulnerabilidad máxima, la decisión de sostener mejoras continuadas sobre los sectores económicamente más postergados impactó en un efecto de distribución vertical de ingresos de arriba hacia abajo, desvirtuando la estructura jerárquica original del sistema previsional. De hecho, para 2006 alrededor del $65 \%$ de los jubilados cobraban el haber mínimo, reconvirtiendo la pirámide previsional en una figura con "una inmensa base y ladrillos superiores bastantes estrechos"(Zaiat, 2006) $\underline{22}$. Por su parte, las leyes de Inclusión Previsional -que es lo que específicamente ordena este trabajoterminarían de tensionar esta figura piramidal. Al flexibilizar los criterios de acceso, harían que la base se vea inmensamente abultada por millones de nuevos perceptores de la jubilación mínima. Los PIP incorporarían-en igualdad de derechos y prestaciones respecto de los jubilados por los carriles tradicionales - a millones de personas que, o bien venían de trayectorias intermitentes en el mercado formal de trabajo, o bien venían de la inactividad económica. Como resultado de la primera ley de fines de 2004, se alcanzaron niveles de cobertura prestacional casi universales, valores que por cierto resultaron inéditos en nuestra historia y únicos en la región. La segunda ley de moratoria buscaría explícitamente sostener estos parámetros.

De este modo nos encontramos con un sistema previsional que, después de casi un siglo de existencia, trastocaría por diversos dispositivos sus lógicas de funcionamiento tanto en lo que hace a los aspectos cuantitativos como cualitativos. A riesgo de ser redundantes, queremos destacar la gravitación de dos aspectos recién mencionados. En primer lugar, el hecho de que los valores alcanzados de casi universalidad de cobertura de las personas en edad de jubilarse construiría una categoría de sujetos con derecho, evidentemente mucho más amplia que los trabajadores formales. En segundo que, por diversos mecanismos, el sistema previsional terminaría generando procesos distributivos que mejorarían las condiciones de los adultos mayores con trayectorias más desfavorecidas. En la configuración de ambas dimensiones los PIP fueron herramientas clave. Y, por eso, la centralidad que le damos en este artículo.

\section{¿Pero qué fueron los Planes de Inclusión Previsional?}

Los Planes de Inclusión Previsional se insertan, como ya dijimos, dentro de un conjunto más amplio de políticas que se incluyen en lo que Danani y Beccaria (2011) y Danani e Hintze (2011y 2013) denominan una contra-reforma .En sí mismos, fueron una política que surgió de una Ley (la 25.994) de escasa visibilidad pública $\underline{23}$, nacida de un acuerdo entre bloques y aprobada sin 
discusión en el Congreso, en el marco de una coyuntura considerada de emergencia y que fundamentalmente consistía en la implementación de dos medidas principales -la jubilación anticipada y una moratoria previsional- que no eran en sí mismas novedosas.

Los PIP fueron aplicados en dos instancias; la primera se inició con la recién mencionada Ley $\mathrm{N}^{\circ}$ 25.994, que fue sancionada a fines de 2004, puesta en marcha en enero de 2005 y estuvo vigente hasta abril de 2007. Para Rofman y otros (2010) el proceso del cual surgió esta política fue a "puertas cerradas", donde pocas personas, excluyendo en general los referentes principales del tema, decidieron los dispositivos a implementar. De allí su poca visibilidad pública. Por su parte, las expectativas iniciales sobre esta ley-que ni siquiera era nominada como Plan de Inclusión Previsional $\underline{\underline{24}}$ no iban más allá de las propias de una acción administrativa de relajamiento de las condiciones de acceso (Dvoskin, 2012b).El instrumento principal emanado de aplicación era la propuesta de la jubilación anticipada $\frac{25}{}$, aunque incluía un artículo que permitía una moratoria con especiales facilidades de acceso. Una vez vigente, el artículo de la moratoria fue cobrando fuerza propia.

De este modo, la Ley25.994, llamada inicialmente de Jubilación Anticipada, no abordaba cuestiones estructurales del paradigma previsional vigente, sino que se presentaba como un instrumento burocrático, de intervención puntual, excepcional y cortoplacista sobre una serie de condiciones sociales que se entendían absolutamente desbordadas. Sin embargo, su impacto excedió ampliamente las expectativas iniciales. Por un lado, por su inmensa extensión de cobertura $\underline{26}$. Pero además, esta primera moratoria establecería dos parámetros fundamentales en la reconfiguración de los criterios de legitimación del derecho previsional. El primero, el acceso sin restricciones a todos aquellos que, cumplida la edad necesaria en 2004, no tuvieran la totalidad de aportes requeridos 27 . El otro, el hecho de que para inscribirse a la moratoria no fuera necesario más que el pago de la primera cuota $\underline{28}$. Con esto, se accedía a la jubilación y a todas sus prestaciones conexas $\underline{29}$ en igualdad que el resto de las personas jubiladas. Valga como mención el hecho de que, una vez finalizado el pago de las cuotas por moratoria, no quedaría rastro visible $\underline{30}$ que evidenciara la diferencia en la modalidad de acceso al sistema respecto a cualquier otra persona jubilada con haber mínimo. Así, no sólo la primera instancia del PIP incorporó a más de 2.5 millones de nuevos jubilados y jubiladas $\underline{31}$, sino que además $-\mathrm{y}$ a diferencia de la tendencia regional a la aplicación de políticas focalizadas de transferencia de ingreso para la vejez-se trató de una política con efectos universalizantes e igualadores sin precedentes $\underline{32}$.

Hacia fines de 2014 se reabriría el debate respecto a la necesidad de nuevas medidas específicas de acceso a la jubilación porque, a pesar de la recomposición socioeconómica vigente por más de 10 años, los persistentes límites del mercado de trabajo harían que cíclicamente personas que iban alcanzando la edad jubilatoria quedaran excluidas de la prestación previsional. Para 2010, el INDEC comenzaría a registrar un paulatino decrecimiento de la tasa de cobertura previsional de su pico histórico de $95.9 \%$ de población adulta mayor. Y esto evidenciaba que la moratoria no había sido más que una medida temporalmente acotada para un problema que era estructural.

La Ley 26.970 daría cuerpo a la Segunda Etapa del Plan de Inclusión Previsional $\underline{33}$, vigente entre 
septiembre de 2014 y octubre de 2016. En este caso sí se daría una amplia difusión pública, tanto desde el gobierno como desde los medios de comunicación que le dieron un masivo tratamiento al tema. Esta segunda etapa se diferenciaría especialmente de la primera al incluir una serie de restricciones de acceso, focalizándose ahora los límites de la inclusión previsional hacia poblaciones consideradas socioeconómicamente vulnerables. Esto merece un comentario:

Como resultado de la primera etapa del PIP, se registró un importante número de personas jubiladas por moratoria que no necesariamente provenían de condiciones de vulnerabilidad socioeconómica. El carácter universalizante que tuvo la medida había habilitado situaciones de igualdad a personas que arrastraban trayectorias de informalidad laboral, desocupación o inactividad económica, fuere cual fuere su posición en la estructura social. En ese marco, circularían distintas críticas respecto de aquellos que habían recurrido a las facilidades de la moratoria sin tener "necesidad" $\underline{34}$. Esta misma

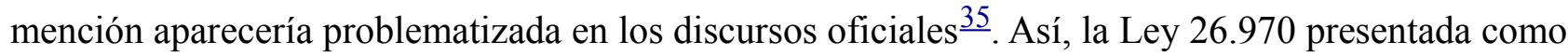
continuidad-pero también como corrección de la anterior moratoria- se haría eco de este asunto, incluyendo como requisito que los destinatarios completaran una evaluación social de su situación patrimonial y de ingresos.

Recapitulando, los PIP aparecen como una pieza clave dentro del proceso más amplio de intervenciones en materia previsional en la primera década y media del siglo XXI. A lo largo de todo ese proceso, aunque especialmente en el momento de la reestatización del sistema, fueron explicitados algunos de los ejes que articulan el debate político cultural respecto a este tema. Danani e Hintze (2011) destacan allí la puesta en agenda de los valores de solidaridad como principio rector, de redistribución e inclusión social como objetivos concretos, y de rejerarquización del papel de la intervención estatal como agente igualador, proveedor de certezas y seguridades a las personas.

En particular, en lo que atañe a las discusiones públicas abiertas por los PIP, encontramos un pasaje de una primera etapa de escasa atención hacia los mismos $\underline{36}$, a un segundo momento -en el marco de la Segunda Ley de Inclusión Previsional- de alta exposición mediática de los debates relacionados al tema.

Para la Segunda Moratoria las noticias permearán más claramente el posicionamiento del gobierno nacional -que en buena medida será asumido como propio por el grueso del arco político-, destacándose en estas nuevas narrativas la explicitación del argumento del derecho de las personas mayores a la seguridad social y el reconocimiento de la búsqueda de universalidad de la cobertura como un valor socialmente compartido. En esta etapa el discurso estará mucho más anclado en la comunidad toda, como actor responsable de la protección social de sus miembros mayores, recuperando una gramática de tipo bienestarista y ciudadana. Por su parte, también habrá mucha información disponible sobre los procedimientos de acceso a la moratoria, mostrando la sencillez y disponibilidad universal de su tramitación.

Sin embargo -y paradójicamente-, al tiempo que se despliega una fuerte presencia discursiva de la noción de derecho de todo envejeciente a ser socialmente sostenido, la nueva modulación narrativa incluiría una contradicción sustancial al introducir un criterio de focalización. De modo que lo que se enunciaría por primera vez como un derecho para todos y todas (y ya no solo para los 
trabajadores formales), a la vez sería nombrado como una política accesible sólo a aquellos que demostraran condiciones de "necesidad".

\section{Entre los hechos y los dichos. Los PIP significados en primera persona}

Si hasta aquí hemos visto la estructura del sistema previsional de nuestro país, puntualizando especialmente en los PIP y la transformación que generaron en su extensión y composición, y cómo esto provocó que millones de personas anteriormente excluidas del derecho a la seguridad social pasaran a ser consideradas sujetos con nuevos (e iguales) derechos, ahora vamos a detenernos en la pregunta por la construcción argumental de esta experiencia en primera persona.

Los datos que presentamos surgen de un trabajo de campo realizado en el ámbito de la ciudad de La Plata entre los años 2014 y 2015, y que constó de entrevistas en profundidad a destinatarios de la primera y segunda moratoria. Aunque sus resultados carecen de valor estadístico, la emergencia reiterada de algunos tópicos nos permite formular interrogantes acerca de algunas dinámicas entre experiencias de inclusión y lógicas de significación.

He aquí algunos de los elementos más salientes de esa investigación:

\section{Las vías de llegada a la Moratoria y los argumentos legitimantes}

Las trayectorias vitales de nuestros entrevistados dieron cuenta de diversidad de caminos recorridos, aunque todos tuvieran como denominador común la falta de aportes previsionales que garantizaran el acceso a la jubilación por otros carriles. En todos los casos las personas aquí investigadas tuvieron -en algún momento de sus vidas- experiencias de actividad económica extradoméstica. Para las mujeres se repetirían períodos de inactividad asociados a los ciclos reproductivos y, en casi todos los casos, aparecerían experiencias de informalidad laboral o cuentapropismo. Nos llamó la atención por su parte, la ausencia de referencia directa a experiencias de desempleo $\underline{37}$.

Así, trayectorias laborales intermitentes e informales arrojaron a estos sujetos a la falta de aportes. Y los aportes constituyeron el vector principal de acceso que institucionalmente ordenó la moratoria. Su ausencia fue el motivo que justificó la política en sí. Su cálculo y el pago en cuotas, la credencial de inclusión.

Es decir, la esencia de este dispositivo político supuso la cancelación -a través de la moratoria en cuotas- de los aportes adeudados. $\mathrm{Y}$ encontramos que estas cuotas se convirtieron en algunos casos en argumento de merecimiento y reversión de posibles estigmas. Nos diría Graciela: "Mirá, yo creo que a nosotros no nos están regalando nada. Porque a nosotros nos están descontando. Vos estás pagando. (...) No lo pagaste en tiempo y en forma, pero lo estás pagando ahora. Y entonces, me parece que no estás perjudicando a nadie" $\underline{38}$.

Esta centralidad otorgada en las entrevistas al aporte como eje del derecho al acceso a la protección social en la vejez tiene coherencia, como ya vimos, con el principio de legitimidad que el sistema previsional sostiene desde su propia formulación originaria. Volveremos sobre este punto más adelante. 


\section{Acerca de los ámbitos de circulación de noticias y debates}

En lo que respecta a los ámbitos de construcción y circulación de información y debates sobre lo previsional, preguntamos acerca de las experiencias de participación o inserción comunitaria de estos sujetos. Nos interesaba saber dónde y en qué interacciones construían sus saberes y significaciones al respecto. Lo que hallamos fue una escasa referencia de nuestros entrevistados a integración en ámbitos colectivos a lo largo de sus vidas. Hubo muy pocas menciones a trayectorias de participación barrial o sindical $\underline{39} \mathrm{y}$, en lo referente a lo previsional, no había casi registro de espacios colectivos de debate acerca de las modificaciones recientes. Así, lo que sabían decían saberlo solo "porque sí", porque lo habían sabido "desde siembre". Y no aparecía referencia a confrontación de diversas opiniones, ni espacios de intercambio o deliberación respecto a lo que tenía que ver con el derecho a la jubilación; tampoco acerca de la medida que los implicaba como destinatarios. Hubo solo un grupo de señoras con participación comunitaria, compañeras de un taller de tejido en un Centro de Jubilados, que de todos modos dijeron que ellas no solían hablar de "esas cuestiones". Sin embargo, y en lo que respecta a los PIP, terminarían reconociendo que entre ellas habían funcionado en algunos casos como facilitadoras de información o ayuda en la gestión de los turnos $\frac{40}{}$. Más allá de estas mujeres, el resto manifestaba haberse enterado de la existencia de los PIP por los medios de comunicación, o por el "boca en boca" entre vecinos y familiares, y no recordaba haber reflexionado demasiado al respecto.

Aún menor fue la referencia a posibles debates al preguntarles por su opinión sobre los formatos de funcionamiento del sistema previsional. A pesar del tiempo transcurrido desde la reestatización y creación del SIPA, consultados sobre sus preferencias entre el anterior modelo mixto o el actual de reparto y solidaridad intergeneracional, tenían una gran desinformación de lo que se trataba $\underline{41}$.

En cuanto al conocimiento de la existencia de los PIP como programa, encontramos una diferencia clara entre la primera y la segunda etapa en relación a la disponibilidad de información, publicidad de cómo realizar el trámite y la certeza de que era una prestación real que ya había alcanzado a millones de personas. Los entrevistados mencionan para la segunda moratoria una fuerte presencia de las campañas publicitarias de la ANSES, expresando que ahora sí pudieron hacer los trámites por su propia cuenta $\frac{42}{}$. Es el caso de Marta, que comenta que cuando se jubiló su marido (también por moratoria) acudieron a un abogado. Pero cuando le tocó a ella no fue necesario "porque yo ya había aprendido. Pero encima había un montón de propagandas que te decían cómo hacerlo. Yo lo veía por ejemplo en los carteles de cuando pasaban el fútbol. Y aparte en el ANSES te lo hacían muy fácil”.

Tan fácil resultaría el trámite, que entre todos los entrevistados nos impactó el caso de José. Él manifestaba haberse enterado de la existencia de la moratoria en el mismo momento en que había concurrido a la ANSES a efectuar lo que hasta ese entonces creía sería su trámite normal de jubilación. Sin embargo, la empleada que lo atendió encontró que le faltaban años de aportes $\frac{43}{y}$, en un procedimiento administrativo y en el mismo acto, le realizó el trámite inscribiéndolo al PIP. Este hombre, que decía estar en desacuerdo con la propia moratoria por la que había podido finalmente jubilarse, no parecía relacionar el dispositivo de política pública que lo alcanzaba con su posibilidad de acceso inmediato a la prestación previsional; esto tal vez porque nunca llegó a darse cuenta de su situación de potencial exclusión de ese derecho. Tan en desacuerdo estaba, que repetidamente se 
expresó disconforme con los descuentos que mensualmente le realizaban en el haber (la cuota de la moratoria), a los que él calificaba como "un robo del gobierno".

En síntesis, si bien hay diferentes trayectorias de llegada a la moratoria entre nuestros entrevistados, hay de todas maneras algunas similitudes. La falta de aportes es la más evidente; aunque también aparecerá su escasa inserción colectiva-comunitaria y una poca gravitación de otros discursos referidos a la jubilación que no sean los provenientes de sus ámbitos de sociabilidad doméstica, o de los medios masivos de comunicación. Esto, incluso, respecto a lo propio del programa en el que ellos resultarían incluidos.

\section{La Jubilación como derecho... ¿de quién es?}

La cuestión de la jubilación como un derecho fue el tópico más insistentemente recorrido en las entrevistas. En gran medida, porque la investigación partía de la visualización de los PIP como una política pública con capacidad concreta de extender masivamente derechos a quienes anteriormente no los tenían. Por otro lado, porque la segunda etapa del PIP había puesto en locución una estructura argumentativa en la que, a la vez que la jubilación era presentada como un derecho de todos y todas las envejecientes, y alcanzar la universalidad de su cobertura era un objetivo declarado del Estado, se incluía un mecanismo de focalización que restringía esa posibilidad solo a algunos.

Encontramos registro de esta tensión en nuestros entrevistados. Así, en los relatos el derecho a la jubilación podía estar al principio asociado indistintamente a factores como la edad, el trabajo realizado "a lo largo de la vida", o el argumento del derecho otorgado por los aportes, que fundamentalmente terminaría sobresaliendo. Marta expresa una frase que se reiteraría: "Claro que tengo derecho a jubilarme. Si yo trabajé toda mi vida". Pero, al profundizar el diálogo, el peso de los aportes primaba en todos los relatos como elemento principal de legitimación. Y esto, a pesar de que éste había sido justamente el punto débil de cada uno de los sujetos que investigábamos.

Destacamos en este punto un elemento emergente del trabajo de campo que no habíamos contemplado en nuestras formulaciones iniciales. Las entrevistas dieron cuenta de una suerte de sentido común sedimentado acerca de la necesidad individual de cada uno de construir, a lo largo de la vida, estrategias de prevención de las contingencias de la vejez. Una señora lo llamó “educación cívica", dando pistas acerca de cómo se concibe, bajo esta mirada, la idea de comunidad y de ciudadanía en clave individual $\stackrel{44}{ }$. En esta lógica, el aporte (hecho por un empleador, o como aporte autónomo para quienes tenían empleo informal, trabajaban por su cuenta, o eran inactivos) irrumpió en los relatos como una obligación que los sujetos debían afrontar por sí mismos, más allá de las condiciones estructurales en las cuales se insertaran las oscilaciones de sus recorridos de vida.

Las trayectorias laborales que nos encontramos arrojaron casos de relación de dependencia por períodos prolongados, de itinerancia entre la actividad y la inactividad, de intermitencia entre el mercado formal y el informal de trabajo. Las trayectorias de aportes no siempre siguieron esas mismas dinámicas. Sus propias experiencias dejan en claro que trabajo y aportes son dos categorías diferentes. Sin embargo, en la construcción discursiva, muchas veces aparecían como superpuestas o intercambiables, hilvanando cadenas de argumentación que se tornaban ambiguas. Alternativamente, estos sujetos aducían tener derecho a su jubilación por el mero hecho de ser 
viejos, o por haber "trabajado toda la vida"; aunque al mismo tiempo, dejaban entrever que fueron ellos quienes - por uno $\mathrm{u}$ otro motivo- no pudieron evitar caer en las propias condiciones de exclusión de ese derecho. No aportar aparece señalado como una falta que, por imprudencia, por poca capacidad de prever el futuro, o por circunstancias familiares que no pudieron salvarse, han cometido individualmente las personas que hoy llegan a la moratoria. La lectura era en general de autoculpabilización. Parafraseando a W. Mills (1964), a estos jubilados y jubiladas les resultaba muy difícil insertar los vaivenes de sus propias biografías en los recorridos sinuosos de la historia de la estructura socioeconómica de nuestro país.

Saliendo de la cuestión del trabajo y los aportes, aparecieron otros elementos que sumaron opacidad a la concepción de la jubilación como derecho de todos y todas. Y es que la repregunta obligaba a los entrevistados a desnaturalizar algunas de las afirmaciones que espontáneamente surgían. Ese fue el caso de Víctor, quien incorporó dentro del campo de delimitación de lo que él consideraba sujetos con derechos la cuestión de la nacionalidad o la identidad de género. La pregunta por quiénes eran aquellos que él inicialmente había identificado como "todos" lo hizo cuestionarse si los extranjeros, por ejemplo, debían quedar incluidos al derecho a la jubilación. Pero mucho más explícito fue cuando este mismo hombre, en un tono a medio camino del chiste, dijo: "iBueno, pero ahora no me vas a venir con que un travesti también va a tener derecho a jubilarse!".

\section{La moratoria como cuestión de género ¿y de necesidad?}

Más allá de las muchas marcas de sentidos asociables a una construcción simbólica de largo plazo en relación a lo previsional, surgió en las entrevistas un aspecto propio de los efectos de los PIP que sí podríamos pensar como radicalmente novedoso: la presencia de los debates respecto al derecho de las mujeres a tener derecho a la jubilación.

$\mathrm{Y}$ es que sin que fuera un resultado intencionalmente buscado, la primer moratoria tendría un impresionante impacto de género. Según la ENAPROSS $\underline{45}$ (2011) el 88\% de las altas de la primera moratoria correspondieron a mujeres $\frac{46}{}$. A tal punto se daría el proceso de feminización de la moratoria, que por un tiempo se la denominaría coloquialmente como "Jubilación de Amas de Casa" dando pistas no sólo de una cuestión de género, sino también de la inclusión $-y$ reconocimiento social por defecto- de un tipo de actividad tradicionalmente excluida de los alcances de la protección social $\underline{47}$.

Esta inclusión, como veremos inmediatamente, fue significada a través de múltiples ambigüedades discursivas, pero indiscutiblemente resultó un tema sobre el que nuestros entrevistados (mayormente entrevistadas) tuvieron una opinión formada. Así, en muchos tramos de la investigación, apareció con naturalidad el debate acerca de la consideración del trabajo reproductivo-doméstico como trabajo que debía ser reconocido. Comenta Graciela en ese sentido: "Sí, como el ama de casa que trabajó toda la vida y sin embargo nunca tuvo derecho a jubilarse. ¿Quién le iba a pagar los aportes? ¿El marido? Entonces yo veo bien esta medida, porque antes no me valoraban a mí...”.

En esta cita se retoma -junto con el reconocimiento de la moratoria como medida que valora socialmente el trabajo dentro del hogar- la centralidad de los aportes como credencial de derecho de 
acceso a la jubilación. Así entonces, la moratoria ¿reconoce y valora la tarea? ¿u otorga autonomía para saldar aportes sin depender del marido? Tal vez Graciela quiso decir un poco de ambas cosas, en el marco de una expresión que demuestra que el sistema previsional está pensado en un tipo de organización social que no sólo reproduce la subordinación de las mujeres, sino que además supone un modelo de arreglo institucional-familiar que hoy está en crisis.

Pero esta no sería la única tensión en el discurso sobre género; la otra aparecerá entrecruzada con la consideración de lo socialmente establecido como el deber ser de las políticas sociales -y de las previsionales en su interior-.

La incorporación de millones de mujeres a la cobertura previsional sería un dato explícitamente abordado-y reivindicado como positivo- en los discursos públicos en torno a la segunda moratoria. Dice la entonces presidenta Fernández de Kirchner al enviar el proyecto de Ley al Congreso -y en alusión a la primer etapa del PIP-: "Esta inclusión previsional no solamente redujo la pobreza, la indigencia entre los adultos mayores, sino también fue una equidad de género, porque el 73 por ciento de esas destinatarias fueron precisamente mujeres, nosotras las explotadas de siempre también".

Ahora bien, en ese mismo proyecto de Ley se implementarían criterios de focalización que ya mencionamos y que afectaban especialmente a las mujeres. Así, se construiría una cadena argumental ambigua donde la popular denominación "Jubilación de Amas de Casa" con que se había conocido coloquialmente a la Primer Moratoria podía a la vez implicar el reconocimiento social del trabajo reproductivo; o bien constituirse en insumo de una mirada crítica respecto de aquellas personas-mujeres sobre todo- que se supondría provenían de la inactividad económica y que habrían aprovechado la moratoria a pesar de no tener "necesidad". Nelly sintetiza en sus palabras esta tensión que aparecía omnipresente en gran parte de las entrevistas:

Porque todos si llegamos a esta edad, por algo llegamos. Y debemos tener un derecho. (...) Entonces, lo mismo para el ama de casa, que tiene que tener derecho(...) Pero, yo veo bien que ahora la moratoria sea solo para los que tienen necesidad (...)Porque ya te digo, el que puede pagarlo por su cuenta también tiene derecho a jubilarse, pero no sacarle a otro pobre que se quiere jubilar. ¿No es así? Pienso, no sé (...) y hubo muchas mujeres que no lo necesitaban y se anotaron en la moratoria.

Así, entre las citas de Graciela y Nelly, queda en evidencia el nudo gordiano de la tensión en la cuestión de género de los PIP. Partiendo del reconocimiento de una desventaja de género estructural (y transversal a los distintos sectores sociales) en la organización social del trabajo, y por tanto en las posibilidades del acceso a la cobertura previsional: ¿cuáles mujeres requerirían de dispositivos específicos que facilitaran su jubilación? La segunda moratoria, el grueso de la opinión pública, y Nelly, dirán que aquellas que puedan demostrar condición de necesidad socioeconómica como carta de merecimiento.

\section{Conclusiones}

Recorrimos en este artículo diversas dimensiones de las políticas previsionales, comenzando con su configuración originaria y analizando algunas de sus transformaciones a lo largo de su siglo de 
historia. Especialmente hicimos hincapié en las profundas transformaciones que derivaron de la implementación de los PIP, una política que duplicó en muy poco tiempo la capacidad de cobertura del sistema previsional. Los Planes de Inclusión Previsional fueron estudiados dentro de una batería más amplia de políticas en la materia, que fue a su vez contextualizada en un clima de época marcado por la crisis socioeconómica y política de fin de siglo pasado y la reivindicación de la intervención estatal y la resemantización de las políticas sociales en clave de derechos y universalización. Allí, las moratorias aparecieron como un dispositivo de intervención pública con una capacidad radicalmente inclusiva e igualadora. Sin embargo, venimos viendo que esta política parece haber tenido menos potencialidad, a la hora de pensar el despliegue de sentidos novedosos acerca de las matrices de ciudadanía y pertenencia social.

En términos de las significaciones típicas de lo previsional, señalamos la existencia de algunos sentidos comunes sedimentados a lo largo del tiempo, que funcionarían como valores inamovibles de lo socialmente esperable en torno a la jubilación. Especialmente, la concepción de la protección social en la vejez como un derecho derivado de las trayectorias laborales por un lado; aunque también -y en modo superpuesto y no necesariamente como parte del mismo proceso- de la capacidad de aportes a lo largo de la vida.

. En ese marco, las propias lógicas de diseño de los PIP (la moratoria) y las enunciaciones que con el tiempo se fueron construyendo a su alrededor, derivaron en una serie de tensiones en las que, aunque explícitamente flexibilizados, persistían y se reafirmaban los criterios típicos de lo previsional. Estas tensiones aparecieron en el trabajo de campo, donde la mayoría de nuestros entrevistados -todos sujetos que accedieron a su jubilación a través de esta política públicasostendrían argumentos de legitimidad de su derecho, atados en última instancia a los mismos criterios de lo laboral y los aportes que anteriormente los habían mantenido excluidos.

La escasez de discursos sociales que pusieran en alocución otros sentidos posibles sobre lo previsional potenció aún más esta ambigüedad típica de las moratorias. Al inicio la moratoria fue asumida como una medida de corte administrativo burocrática. Un relajamiento de los requisitos que, en su esencia, no pretendía cambios estructurales. El impacto de su implementación desbordó lo esperado y transformó a esta medida -originalmente marginal- en una política de gran relevancia. A partir de la campaña presidencial de 2007, y más aún en el marco de la estatización de las AFJP a fines de 2008 y en el posterior lanzamiento de la Asignación Universal por Hijo en 2009, el kirchnerismo construiría-desde una reivindicación ex post de los resultados-una cadena argumental que hasta le pondría un nombre a la política pública: Plan de Inclusión Previsional. Ahora sí, se trataría de un discurso inserto en la búsqueda de ampliación de derechos y ciudadanía que, además, relacionaría diferentes políticas sociales dentro de una misma lógica estatal de intervención. Hacia la segunda etapa, los PIP fueron públicamente presentados desde su inicio en esta matriz enunciativa en clave de derechos, identificando explícitamente a la comunidad (o al Estado) como responsable de garantizar la contención universal de sus adultos mayores. Sin embargo, ahora la introducción de restricciones de focalización sumaría otras nuevas tensiones más allá de las cuestiones del trabajo y los aportes. La segunda etapa de los PIP incluiría la dimensión de la "necesidad", como credencial de merecimiento.

De este modo, estaríamos frente a una política pública con gran potencial de transformación 
material, aunque de menor incidencia en la modificación de las matrices simbólicas asociadas a lo previsional. Sin embargo, también aparecerían otras huellas en las voces de los destinatarios. Y es que la inercia de sentidos de lo típicamente entendido como justo en relación al derecho a la jubilación, estuvo atravesada por procesos de resignificación a la luz de las propias experiencias de vida.

A pesar de lo paradójica que podía resultar la persistencia del valor de lo laboral como argumento de derecho (en sujetos con trayectorias de múltiples dificultades de acceso al trabajo), la mención de haber "trabajado toda la vida" sistemáticamente presente en las entrevistas, daba cuenta también de los PIP como instrumento de reconocimiento de un trabajo que antes no había sido socialmente contabilizado como legítimo.

Encontrábamos esto en casi todos los casos, aunque con mucha más fuerza cuando hablábamos del trabajo reproductivo-doméstico. La formulación de la expresión "Jubilación de Amas de Casa" ,como referencia al impacto de feminización de las moratorias, hizo visibles los múltiples procesos sociales desubalternización de las mujeres. Los relatos darían cuenta de la concepción del derecho asociado al trabajo ("como el ama de casa que trabajó toda la vida y sin embargo, nunca tuvo derecho a jubilarse"), pero, también, del dispositivo de los PIP como generador de autonomía ("¿Quién le iba a pagar los aportes? ¿El marido?"). Con la moratoria, las mujeres-con o sin trayectorias extradomésticas - pasarían a ser consideradas trabajadoras, y ellas mismas pagarían sus aportes.

Esta extensión formidable de derechos (que revertiría la estructura previsional tradicionalmente masculina) se frenó con la irrupción de los criterios de focalización de la segunda etapa del PIP. La focalización sumaría otro vector de ambigüedad a esta política y a los discursos recogidos en la investigación. En lo que respecta al género, ahora solo las mujeres, consideradas primero como pobres, serían después potencialmente vistas como legítimas destinatarias de reconocimiento por su trabajo reproductivo.

Por su parte, el eje de los aportes será un argumento que termine teniendo en los relatos, aún más peso que el laboral. En parte porque, como vimos, los aportes son condición estructurante de la moratoria en sí misma. El pago de la cuota subsanaría un aporte que no había sido realizado en tiempo y forma, restituyendo legitimidad a un derecho (y a una obligación) que había sido temporalmente puesta en suspenso. Lo previsional -término que viene justamente de la idea de prever- aparecía en los relatos asociados a la construcción de una suerte de cultura cívica de la responsabilidad individual en la generación de resguardos a futuro: "Era como la Caja de Ahorro, nos enseñaban a ahorrar. Y también te enseñaban lo de los aportes para cuando tuvieras que jubilarte...".Acá había algo profundo en la construcción significante. Emergía, en esta mirada de la responsabilidad incumplida, la sedimentación de un sentido de autoculpabilización.

Las trayectorias de vida de estos sujetos estaban signadas por múltiples momentos en los que no pudieron cumplimentar el mandato de la previsión para su futura vejez. En general, por cuestiones propias del proceso histórico, social y económico que los excedía. Aunque esto no resultaba visible para ellos. La moratoria daba en sí misma cuenta de la necesidad de implementación de medidas sociales para dar solución a problemáticas, que también eran sociales. Pero los sujetos aquí 
indagados no veían sus propias biografías en esta clave. No sólo porque les costaba asociar sus desventuras al ritmo de los procesos socialmente estructurales, sino porque además tampoco se veían a sí mismos como parte de una categoría social que requería de dispositivos de políticas específicas. En especial, esto es visible en José, que ni siquiera estaría de acuerdo con la propia moratoria que lo hacía acceder al derecho a la jubilación. La escasez de participación de nuestros entrevistados en espacios colectivos de debate creemos que potenciaría esta lectura en clave individualizada.

Así, para muchos de nuestros jubilados los PIP aparecieron como una "yapa", un impensado, una oportunidad que no demandaban ni esperaban. Y que cuando surgió, les permitió subsanar las obligaciones que anteriormente no habían podido cumplir por sus propios medios.

Finalmente, ante la tensión latente de la idea de la jubilación como derecho prácticamente universal, encontraríamos en algunos relatos otros nuevos clivajes (además de los relacionados al trabajo y los aportes) que construirían otras fronteras de potencial limitación de acceso. ¿Qué ocurriría con los extranjeros? ¿y con aquellos con identidades de género diferentes? En este sentido, la potencialidad de la igualdad parecería operar como un factor amenazante, incluso en aquellos que, producto de la aplicación de los PIP, habían sido sujetos de radicales procesos de igualación.

Así, creemos que los Planes de Inclusión Previsional generaron posibilidades de extensión de cobertura e igualdad de condiciones de derechos, que habilitaron, a su vez, la puesta en debate de las maneras en la que nuestra sociedad define sus contornos de ciudadanía. Estas discusiones atravesaron las agendas públicas, políticas, académicas, pero también se dieron al interior de millones de hogares alcanzados.

Ya sea por la propia forma asumida desde su diseño, por los discursos públicos movilizados en los distintos contextos, o por la persistencia de matrices de sentido de larga data, los debates abiertos por los PIP dejaron en evidencia un sinnúmero de tensiones acerca de cómo definir quiénes son los sujetos adultos mayores con derecho a ser socialmente sostenidos. Dejaron abierta la pregunta por la comunidad y los márgenes de igualdad que resultan socialmente tolerables al momento de potenciar procesos de inclusión. Y justo cuando esta pregunta afloraba en toda su magnitud, se dio por terminado el ciclo de esta política.

El año 2015 implicó un cambio en la gestión de gobierno, y una redefinición del clima de época, tanto para la concepción de las estatalidades como de los derechos y las ciudadanías. Con la discusión sobre lo previsional a flor de piel, vertiginosamente se avanzó en nuevas reformas que aún no terminan de definirse, aunque auguran retrocesos en múltiples dimensiones.

Diremos, como reflexión final, que el modo en que socialmente retomemos la discusión que quedó pendiente al cierre de la experiencia de los PIP, nos permitirá pensar en profundizar un camino de universalización con aumento de la igualdad; o retroceder nuevamente a las matrices más típicas de la seguridad social estamentada, reproductora de desigualdades y acotada a una pequeña porción de la sociedad. 


\section{Agradecimiento:}

Agradezco especialmente los comentarios y correcciones sugeridas por los evaluadores, que resultaron muy relevantes para un enriquecimiento sustancial de este artículo.

\section{Notas}

1 Las medidas de la jubilación anticipada y las moratorias previsionales englobadas en la categoría de los PIP serán nominadas de ese modo unos años después de entrar en vigencia. Volveremos en detalle sobre esta cuestión en páginas posteriores.

$\underline{2}$ Trabajé esta cuestión de modo más extenso en Corsiglia Mura (2014).

$\underline{3}$ Existe un rico debate en relación a la diferencia que hay entre los conceptos de universalidad y masividad a raíz de la extensión de cobertura de varias de las políticas sociales de la década pasada. Para profundizarlo, puede consultarse De Sena (2011). De todas maneras, a los fines de nuestra afirmación, alcanza con decir que desde la enunciación oficial se sostendría, a partir del año 2007 por lo menos, el objetivo de alcanzar la universalidad de distintas prestaciones. Esto vale para la cobertura previsional, pero también sería argumento central para el caso de la Asignación Universal por Hijo desde que esta fuera implementada en el año 2009.

4 De las cuales 2.675.341 fueron incluidas en la primer moratoria, y 921.243 en la segunda (BESS, 2017).

$\underline{5}$ Sin ser un debate cerrado, existe una interesante producción de literatura específica que sostiene el surgimiento de distintas formas de política social a partir de los primeros años del 2000. Trabajé más en extenso el tema, y el lugar de las modificaciones de las políticas previsionales en ese debate en particular, en Corsiglia Mura (2013).

6 La otra política social que es analizada en esta sintonía en parte de la literatura académica, y que también se inscribiría dentro de la Seguridad Social (aunque no destinada a los adultos mayores) es la Asignación Universal por Hijo.

7 La expresión la tomo de Arcidíacono (2012).

$\underline{8}$ Las diferencias que expresan estos modelos nos remiten a distintos arreglos institucionales de resolución de la protección social. El contributivo refiere fundamentalmente al modelo del seguro social biscmarckiano, cuyo origen tuvo lugar en Alemania a fines del s. XIX y es indisociable del rol de los sindicatos como actores sociales. El segundo, se lo relaciona en su versión original al planteo que Beveridge hiciera al parlamento inglés en el marco de la segunda posguerra, de aplicación de un régimen de protección universal de protección social que asegurara un piso mínimo de cobertura independiente de la contribución realizada.

$\underline{9}$ El criterio del 82\% móvil -reclamo histórico de trabajadores en actividad, de jubilados y de parte de los discursos de una fracción importante del arco político- opera como imaginario consolidado de lo que debería ser la fórmula de actualización de haberes. Lo que este criterio establece no es otra cosa que la traslación al mundo pasivo de las segmentaciones salariales del mundo activo, 
postulando la necesidad de sostener socialmente, al momento de la jubilación, las distintas jerarquías relacionadas con el sector de actividad de cada rama. Para un debate más profundo sobre este tema puede consultarse Dvoskin (2012a).

10 En 1904 se crearía la primer Caja de Jubilaciones para empleados públicos. Ley 4349.

11 Hay que destacar que en este momento todavía se trataba de un sistema joven con escasa cantidad de personas efectivamente jubiladas.

12 Los picos de cobertura previsional del período previo a la aplicación de los PIP serán entre los años 74 y 80, que superan el 70\% (Arza, 2004, citado en Lo Vuolo 2007, p. 513).

13 En el año 1994, en el marco de las políticas neoliberales del Consenso de Washington, se transformó el sistema previsional argentino hacia un régimen mixto, donde coexistiría un subsistema de reparto con otro de capitalización individual (Ley 24.241). Claramente, la reforma estaría sesgada hacia este último. Y de hecho, hacia principios del 2000 , casi el $90 \%$ de los aportantes lo hacían al Régimen de Capitalización (Lo Vuolo, 2007, p. 520). Esta reforma se contextualizó en los postulados de la Nueva Ortodoxia Previsional, que impulsó similares reformas en otros diez países de América Latina (Lo Vuolo, 2007, p. 518).

14 A partir de la reforma de 1994 se cambió la edad jubilatoria (pasando de 55 a 60 años para las mujeres y de 60 a 65 para los hombres) y se aumentó la cantidad de años de aportes requeridos, elevándose de 15 a 30. También se modificó la fórmula en el cálculo de haberes. (Lo Vuolo, 2007, p. 518).

15 Danani y Beccaria (2011) explican que la "cobertura horizontal", es decir, la proporción de personas en edad de jubilarse y que efectivamente accede a la prestación, cayó aproximadamente un $10 \%$ entre 1996 y 2002.

16 El envejecimiento de la población es un tema que viene siendo especialmente atendido en el mundo y en la región. Está relacionado con la transformación de largo plazo de las pirámides sociodemográficas. Argentina tiene una de las estructuras demográficas más envejecidas de América del Sur y, de hecho, según datos del Censo 2010 la proporción de población de 60 años o más registra una tasa del orden del 14\%. Esto sitúa a nuestro país en la categoría de "país envejecido" (Roqué y Fassio, 2012).

17 Es necesario desatacar la existencia de un sistema de cobertura paralelo -consistente en pensiones de tipo no contributivo- vigente al menos desde el primer peronismo (Arza y Chahbenderian, 2014). Pero este sistema de administración diferencial, tuvo una existencia marginal respecto del sistema previsional en general.

18 A pesar de que la categoría de exclusión es controvertida -como bien da cuenta de ello Castel ([1995] 2010, p. 257)- la perspectiva de Fleury nos permite tomar dimensión del lugar de no acceso a derechos de estos adultos mayores que quedaban a la intemperie de la seguridad social.

19 Serán 14 aumentos por Decreto del PEN, hasta que en 2008 se sancione la Ley de Movilidad Jubilatoria. 
20 El porcentaje diferencial de aumento fue escalonado entre 2002 y 2007, disminuyendo a medida que los haberes eran más altos. Al principio solo hubo aumentos para los haberes mínimos, a partir de 2004 se incorporó, con menores tasas, a jubilados por encima de la mínima; pero particularmente la escala más alta de haberes no recibiría incrementos hasta el año 2006, perdiendo distancia respecto de los estratos inferiores de la pirámide. Esto será uno de los motivos de litigiosidad y de pronunciamientos sistemáticos de la CSNJ desde el año 2005, y será asimismo una de las cosas que se busquen revertir a partir de la Ley llamada "de Reparación Histórica" de 2016. A partir de 2008, entraría en vigencia la Ley de Movilidad Jubilatoria con un índice de actualización único para todos los tramos.

$\underline{21}$ Ley que permitía que aquellos inscriptos en el sistema de las AFJP pudieran retornar, si así lo deseaban, al sistema estatal y de reparto. Es necesario recordar que este movimiento estaba taxativamente prohibido anteriormente.

$\underline{22}$ Es necesario recalcar que este proceso era resultado de un crecimiento de abajo hacia arriba, y no de una masificación del haber mínimo por caída generalizada en el ingreso.

$\underline{23}$ Las notas periodísticas dedicadas en ese momento al tema son escasas y no cuentan con más que uno o dos párrafos en los que se detalla las condiciones del proyecto.

24 Siguiendo a Dvoskin (2012b) encontramos que las primeras enunciaciones en términos de Programa de Inclusión Previsional aparecerán recién a fines de 2006, refiriéndose principalmente a los convenios entre el gobierno nacional, los gobiernos provinciales y los municipios, para que estos últimos lleven adelante las campañas necesarias para acercar a los adultos mayores a las oficinas de la ANSES. Posteriormente, a partir de la lectura de los resultados de la implementación ya en curso -y en el marco de un año de campaña electoral como fue 2007- aparecerán más referencias a las moratorias y comenzará a aparecer públicamente el nombre de Programa de Inclusión Previsional o, alternativamente, el nombre de Programa de Inclusión Social al Sistema Previsional.

$\underline{25}$ Destinada a personas que tuvieran la cantidad de aportes necesarios pero que les faltara hasta cinco años para cumplir la edad jubilatoria.

26 Mientras las estimaciones iniciales, según los dichos de los funcionarios en los medios de prensa, esperaban entre 20 y 70 mil inscriptos, la primera moratoria obtuvo entre 2006 y 2007 un pico máximo de altas de jubilaciones que rondaría los dos millones y medio de inscriptos.

27 Podían acceder al beneficio quienes, teniendo la edad cumplida hasta el 31 de diciembre de 2004, no registraban aportes al sistema o los mismos eran insuficientes, o quienes cumpliendo la edad en cualquier momento completasen los aportes anteriores al 30 de septiembre de 1993 (Calabria y otros, 2012, p. 290).

$\underline{28}$ El sistema implementado permitió prorratear la deuda de aportes previsionales en hasta 60 cuotas deducibles del haber mensual, garantizando accesibilidad universal a la medida, ya que no requeriría que los aspirantes tuvieran capital inicial para la cancelación de la deuda.

$\underline{29}$ Entre ellos: igualdad del monto del haber previsional con respecto a los jubilados de la categoría más baja (una vez canceladas las cuotas de la moratoria), mismo criterio de actualización que el resto de los jubilados, acceso en absoluta igualdad de condiciones a la cobertura de la obra social 
Pami, y a la Tarjeta Argenta.

$\underline{30}$ Al menos no en los recibos de haberes, en los padrones de Pami, en el tipo de prestación accedida, etc.

$\underline{31}$ Ver nota al pie $n^{\circ} 4$ de este mismo artículo.

$\underline{32}$ Cabe destacar que en nuestro país también hubo políticas destinadas a adultos mayores asociadas a la lógica de política social focalizada de transferencia de ingresos. Algunas de ellas fueron previas y otras contemporáneas por momentos a la experiencia de implementación de la primer etapa del PIP. Podemos mencionar en ese sentido a las Pensiones No Contributivas (PNC) a la vejez que como ya hemos dicho, son una institución que existe desde larga data, pero que varía en un crecimiento exponencial de su cantidad de beneficiarios a partir del año 2003 ante el levantamiento de la restricción de "altas por bajas"(Arza y Chahbenderian, 2014, p. 14). Sin embargo, el crecimiento de las PNC tendrá su pico máximo en 2006 (alcanzando a un 2.5\% de la población de 65 años o más). A partir de allí, la cantidad de destinatarios cubiertos comenzaría un proceso de declive. Por su parte, en el año 2002 se estableció el Plan Mayores como una suerte de subcomponente del Plan Jefes y Jefas de Hogar Desocupados. A través de éste se otorgaba un beneficio mensual de \$150a las personas mayores de 70 años sin ingresos fijos. Este programa tuvo un alcance limitado. Con el cambio de gobierno en 2003 se lanzó un nuevo plan para los adultos mayores sin ingresos, el Programa para el "Adulto Mayor Más” (PAMM), que articularía con el Plan Familias y que tampoco tuvo un despliegue demasiado relevante. Para mayor información sobre estos programas específicos, y sobre las políticas de transferencia condicionada de ingresos en la región, puede consultarse Arza y Chahbenderian (2014). En cualquiera de los casos, podría sugerirse que la aparición de los PIP mejoraría la oferta en relación a la que brindaban estas políticas puntuales, abriendo la posibilidad a una prestación de mayor calidad, a la vez que expandiendo los límites a una población potencialmente destinataria que no se presentaba como demandante de planes sociales.

33Ahora sí encontramos que esta fue la manera en que se lo denominó por parte del Poder Ejecutivo Nacional al enviarse el Proyecto de Ley al Congreso, presentándolo como parte de una secuencia que retrospectivamente incorporaba a la primer Ley 25.994 como parte del mismo Plan.

$\underline{34}$ Este tipo de formulaciones se encuentran con facilidad en los medios de comunicación y, como veremos, también aparecieron en el trabajo de campo de la investigación que aquí se reseña. Entendemos que el sentido que expresan está anclado, en última instancia, en la inercia de una concepción de las políticas sociales tributaria de las lógicas neoliberales, que consagraron a las mismas como políticas preferentemente focalizadas $\mathrm{y}$ orientadas a atender a poblaciones identificadas como "en riesgo".

$\underline{35}$ Puede verse en el discurso presidencial de anuncio de envío de la Ley al Congreso de fecha $04 / 06 / 2014$.

$\underline{36}$ Es necesario aclarar que, si bien los PIP tienen inicialmente una escasa atención mediática, otros temas referidos a lo previsional ocupan espacios de relevancia en la agenda noticiosa. Entre ellos, y en orden cronológico, lo referido a los aumentos de haberes, los fallos de la CSJN, la Ley de Movilidad y el veto de la Ley del $82 \%$ móvil, la estatización de las AFJP, etc. 
$\underline{37}$ En este punto resultó muy interesante el aporte de algunos colegas que, basados en sus propias experiencias de investigación, abrieron la pregunta a la posibilidad de la subdeclaración del desempleo por posibles prejuicios. Sería ésta una cuestión para mayores indagaciones futuras.

$\underline{38}$ Es interesante en este punto la afirmación de Dvoskin (2017) para quien los aportes realizados a través de la moratoria eran "ficcionales", al no representar la cancelación real de la deuda previsional que mantenían los nuevos jubilados. Más allá de eso, podemos ver cómo los mismos tenían, evidentemente, un alto peso en la justificación que los destinatarios hacían de su lugar de receptores de esta política.

$\underline{39}$ Esto último es consistente con sus trayectorias laborales mayormente informales.

$\underline{40 E s t o ~ r e f i e r e ~ a ~ l a ~ S e g u n d a ~ M o r a t o r i a ~ y, ~ e n ~ e s p e c i a l, ~ d e b e m o s ~ d e s t a c a r ~ q u e ~ a l ~ m o m e n t o ~ d e ~ l a ~}$ entrevista la misma estaba en plena vigencia.

41 Si bien algunos sabían de familiares o conocidos que se habían llegado a jubilar por el Régimen de Capitalización, en general no terminaban de identificar las diferencias con el sistema actual. Uno de los entrevistados, consultado acerca de si sabía lo que eran las AFJP, dijo que recordaba que antes existía en las jubilaciones "algo llamado JP" pero no estaba seguro de qué era.

$\underline{42}$ De los casos aquí observados, en la Primer Moratoria todos recurrieron a la gestión de abogados a pesar de que el trámite no lo requería.

43 José responsabilizaría de esto a uno de sus empleadores, agregando en la entrevista que él desconocía que no le había realizado los aportes por más de cinco años.

44 Dice nuestra entrevistada: "Nosotros antes teníamos educación cívica. Era como la Caja de Ahorro, nos enseñaban a ahorrar. Y también te enseñaban lo de los aportes para cuando tuvieras que jubilarte. Vos sabías que tenías que hacerlo."

45 Encuesta Nacional de Protección y Seguridad, realizada por la Dirección General de Estudios y Estadísticas Laborales, de la Dirección de Estudios y Coordinación Macroeconómica del MTEySS

46 Este dato debe ponerse en contexto con que el proceso de envejecimiento poblacional del que dimos cuenta en la nota al pie $\mathrm{n}^{\circ} 18$, viene de la mano de un complementario y marcado proceso de feminización de la vejez (Roqué y Fassio, 2012). Esto le da aún más relevancia al impacto de la moratoria.

47 Históricamente, el acceso a la protección social (concebida desde el modelo de seguro social, como venimos viendo para nuestro país) identificó como sujeto de derecho al varón proveedor, y eventualmente por derrame, la cobertura se hacía extensiva a la mujer "a cargo", quien se encargaba mayormente de las tareas de trabajo reproductivo-doméstico no asalariado.

\section{Bibliografía Consultada}

Andrenacci, L. y Repetto, F. (2006). Universalismo, ciudadanía y Estado en la política social Latinoamericana. Washington D.C.: INDES. 
Arcidiácono, P. (2012). La política del mientras tanto: programas sociales después de la crisis 2001-2002.Buenos Aires: Ed. Biblos.

Arditi, B. (2007). Ciudadanía de geometría variable y empoderamiento social: una propuesta. En Calderón, F. (coord.), Ciudadanía y Desarrollo Humano. Cuadernos de Gobernabilidad Democrática 1. Buenos Aires: Ed. Siglo XXI.

Arza, C. y Chahbenderian, F.(2014). Pensiones básicas en América Latina. Diseño, cobertura y beneficios comparados en Argentina, Brasil, Bolivia y Chile. Documento de Trabajo del CIEPP, 89.Disponible en: http://www.ciepp.org.ar

Argentina. Ministerio de Trabajo y Seguridad Social de la Nación, Secretaría de Seguridad Social. (2017) Boletín Estadístico de la Seguridad Social (BESS). Primer Trimestre de 2017. Recuperado de http://trabajo.gob.ar/downloads/seguridadSoc/BESS_1trim_2017.pdf

Calabria, A., Calero, A., D'Elia, V., Gaiada, J. y Rottenschweiler, S. (2012). Análisis del Plan de Inclusión Previsional: protección, inclusión e igualdad. Revista de Economía Pública, Social y Cooperativa, 74.

Castel, R. (2010). El ascenso de las incertidumbres. Trabajo, protecciones, estatuto del individuo. Buenos Aires: Fondo de Cultura Económica.

Corsiglia Mura, L. (2013). Estado y políticas sociales en el post-neoliberalismo. Un campo de discursividades en tensión. De Prácticas y Discursos. Cuadernos de Ciencias Sociales, 2(2).

Corsiglia Mura, L. (2014). Estado, discursos en disputa y hegemonías latinoamericanas. Un recorrido a través de la bibliografía regional. Cuestiones de Sociología, 11.

Danani, C. y Beccaria, A. (2011). La (contra)reforma previsional argentina. 2004-2008: aspectos institucionales y políticos-culturales del proceso de transformación de la protección. En Danani, C.y Hintze, S. (coords.), Protecciones y desprotecciones. La seguridad social en la Argentina 19902010. Universidad Nacional de General Sarmiento.

Danani, C. yHintze, S. (2011). Introducción. Protección y seguridad social para distintas categorías de trabajadores: definiciones conceptuales, propuestas de abordaje e intento de interpretación. En Danani, C.y Hintze, S. (coords.), Protecciones y desprotecciones: la seguridad social en la Argentina 1990-2010. Universidad Nacional de General Sarmiento.

De Sena, A. (2011). Promoción de microemprendimientos y políticas sociales: ¿Universalidad, Focalización o Masividad? Una discusión no acabada. Pensamiento Plural, 1, 36-67.

Dvoskin, N. (2012a). Ideas y teorías económicas en los debates sobre la reforma de la seguridad social en Argentina: el caso de la ley de movilidad de haberes previsionales de 1958. Ponencia 
presentada en III Congreso Latinoamericano de Historia Económica y XXIII Jornadas de Historia Económica, San Carlos de Bariloche.

Dvoskin, N. (2012b). De la protesta social a la cobertura no contributiva, y de vuelta a la protesta social: incidencia de las manifestaciones de los noventa en la reforma de la seguridad social de 2005 - 2010. Ponencia presentada en II Jornadas de Sociología Política, UNMDP.

Dvoskin, N. (2017). La reforma previsional del macrismo: el retorno de la estigmatización en la politica social. Épocas, 3; -1-2016; 1-11. Disponible en http://ri.conicet.gov.ar/bitstream/handle/11336/22229/CONICET_Digital_Nro.24889\%20\%281\%29 .pdf? sequence $=2 \&$ is Allowed $=\mathrm{y}$

Fiscella, S. (2005). Estado, ciudadanía y política social. Estudios sobre los sistemas de jubilaciones $y$ pensiones. Buenos Aires: Espacio Editorial.

Fleury, S. (2002). Políticas sociales y ciudadanía. Umbrales,11, 189-215. Disponible en https://www.academia.edu/20696537/Pol\%C3\%ADticas_Sociales_y Ciudadania

Fleury, S. (2006). Democracia, Ciudadanía y Políticas Sociales. Revista Salud Colectiva, 2(2),109112.

Lo Vuolo, R. (2007). Un siglo de previsión social. En Torrado, S. (comp.), Población y bienestar en la Argentina del primero al segundo centenario. Una historia del siglo XX. Tomo II. Buenos Aires: Ed. Edhasa.

Madera, N. (2011). La política de Inclusión previsional en Argentina: análisis y perspectivas. Ponencia presentada en VI Congreso Argentino de Administración Pública, Resistencia.

Mills, C.W. (2003). La imaginación Sociológica. México: Fondo de Cultura Económica.

Mouffe, C. (1998). Ciudadanía democrática y comunidad política. En Laclau, E., Mouffe, C. Torfing, J. y Zizek, S., Debates políticos contemporáneos. En los márgenes de la modernidad. Seminario de Profundización en Análisis Políticos. México: Plaza y Valdes Editores.

Rofman, R., Fajnzylber, E. y Herrera, G. (2010). Reformando las reformas previsionales: en la Argentina y Chile. Revista de la CEPAL, 101.

Roqué, M. y Fassio, A. (2012). Gerontología Comunitaria e Institucional. Módulo2 de la Especialización en Gerontología Comunitaria e Institucional. Universidad Nacional de Mar del Plata.

Zaiat, A. (14 de Mayo de 2006). Deudas a saldar. Página/12. Recuperado de https://www.pagina12.com.ar/diario/suplementos/cash/19-2438-2006-05-14.html 